\title{
ARTICLE
}

Translational Therapeutics

\section{Association of tumour and stroma PD-1, PD-L1, CD3, CD4 and CD8 expression with DCB and OS to nivolumab treatment in NSCLC patients pre-treated with chemotherapy}

Anna-Larissa Nadia Niemeijer ${ }^{1}$, Sara Sahba ${ }^{1}$, Egbert Frederik Smit ${ }^{1,2}$, Birgit Ilja Lissenberg-Witte ${ }^{3}$, Adrianus Johannes de Langen ${ }^{1,2}$ and Erik Thunnissen ${ }^{4}$

BACKGROUND: Immune checkpoint inhibitors are most beneficial in patients with high tumour PD-L1 expression. However, the use of PD-L1 expression is not straightforward. We investigated PD-L1 expression and immune cell (IC) infiltrates in non-small-cell lung cancer (NSCLC) patients treated with nivolumab.

METHODS: Tumour tissue specimens of 139 NSCLC patients were scored for tumour/stromal PD-L1 and various IC expression markers, and associated with durable clinical benefit (DCB) and overall survival (OS).

RESULTS: Median OS was higher for patients with high stromal infiltration of CD8 ${ }^{+}$ICs (9.0 months) compared with patients with low and intermediate infiltration (both 5.0 months, $p=0.035$ ) and for patients with high infiltration of stromal CD4 ${ }^{+}$ICs ( 9.0 months) compared with patients with low and intermediate infiltration (both 5.0 months, $p=0.010$ ) and this was confirmed in the validation cohort. Post hoc analyses showed that biopsies taken after the last line of chemotherapy (ACT) were predictive for DCB and OS, whereas samples obtained before the last line of chemotherapy (BCT) were not.

CONCLUSIONS: Stromal infiltration of ICs can predict response to PD-1-directed immunotherapy in NSCLC patients. Interestingly, we found differences in the predictive value of IC markers between the ACT and BCT biopsies, suggesting that chemotherapy might influence the immune microenvironment.

British Journal of Cancer (2020) 123:392-402; https://doi.org/10.1038/s41416-020-0888-5

\section{BACKGROUND}

The treatment of non-small-cell lung cancer (NSCLC) has been revolutionised by the introduction of antibodies targeting immune checkpoints, including those directed against the programmed cell death 1 (PD-1) or its ligand (PD-L1). ${ }^{1}$ PD-1 is expressed on activated $\mathrm{B}-$ and $\mathrm{T}$ cells and inhibits their functioning upon binding to PD-L1, which is expressed by tumour cells and a subset of immune cells., ${ }^{2,3}$ Nivolumab, an anti-PD-1 checkpoint inhibitor, has clinical activity in patients with NSCLC, and is effective as second-line therapy in patients with advanced-stage NSCLC. ${ }^{4-6}$ Unfortunately, only $\sim 20 \%$ of patients experience a durable response to single-agent nivolumab treatment. ${ }^{4,5}$

Despite promising efforts on tumour mutational burden, PDL1 expression is still the only clinically available biomarker. ${ }^{7,8}$ Nonetheless, the value of tumour PD-L1 expression as a predictive biomarker is not straightforward. There are multiple assays with varying definitions of biomarker positivity, and in patients with the highest expression PD-L1 levels, a positive immunohistochemistry (IHC) test defined by $\geq 50 \%$ tumour PD-L1 expression, the response rate is only $\sim 45 \%$, while those with completely negative $(<1 \%)$ tumour PD-L1 staining still have a response rate of $\sim 10 \%$. $^{9-11}$

There is increasing evidence that immune contexture, i.e., infiltration of tumour tissue by immune cells, plays an important role in the sensitivity of cancers to respond to immune checkpoint inhibitor therapy. ${ }^{12,13}$ Most studies that examined the immune contexture used a mixed cohort of solid cancer patients receiving immune checkpoint inhibitors. Although immune cell PD-L1 expression and $\mathrm{CD}^{+}$infiltration are mentioned as markers correlated with (durable) response, none of the studies agree which predictive marker can be used in NSCLC patients. ${ }^{13-15}$ Therefore, the exact role of these specific immune cells in tumour inflammation and antitumour response to checkpoint inhibition therapy in NSCLC remains uncertain.

We hypothesised that immunohistochemical analysis of the immune contexture beyond tumour PD-L1 expression has predictive value for response to PD-1 checkpoint inhibitor therapy. Using tumour biopsies obtained prior to nivolumab treatment, tumour PD-L1 expression and $\mathrm{CD} 8^{+}$tumour-infiltrating immune cell expression were assessed, as well as stromal PD- $1^{+}, \mathrm{PD}-\mathrm{L} 1^{+}$,

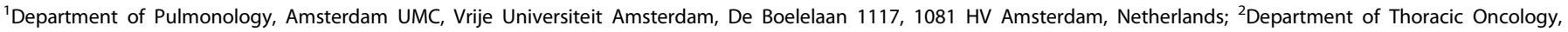

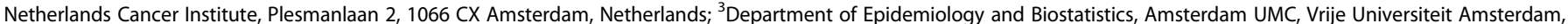

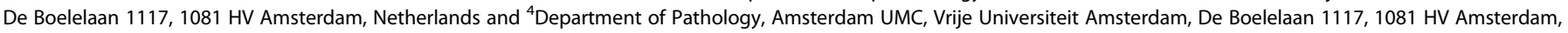
Netherlands

Correspondence: Adrianus Johannes de Langen (j.d.langen@nki.nl)

These authors contributed equally: Anna-Larissa Nadia Niemeijer, Sara Sahba

Received: 3 September 2019 Revised: 30 March 2020 Accepted: 22 April 2020

Published online: 20 May 2020 
$\mathrm{CD}^{+}, \mathrm{CD}^{+}$and $\mathrm{CD}^{+}$immune cell expression. Next, these parameters were associated with durable clinical benefit (DCB) and overall survival (OS) in patients with advanced-stage NSCLC that were treated with nivolumab.

\section{METHODS}

Subjects and samples

Since the introduction of immunotherapy for NSCLC in The Netherlands, patient characteristics, as well as treatment outcome and adverse events, are being prospectively collected in a national lung cancer immunotherapy database for all patients with a pathologically confirmed diagnosis of advanced-stage NSCLC, who are treated with immune checkpoint inhibitors. From this database, we retrospectively selected patients treated with nivolumab (3 mg/kg Q2W) from August 2015 to January 2017 at the Amsterdam University Medical Centers, location: VU Medical Center (VUmc). For each eligible patient, we retrospectively obtained a suitable histological biopsy sample that contained sufficient tumour tissue (i.e., at least 100 tumour cells), and was preferably not decalcified before paraffin embedding. Tissue blocks were obtained prior to the start of nivolumab treatment, either before or after the last line of treatment with platinumdoublet chemotherapy. All tissue analyses were performed at the VUmc Department of Pathology. The protocol was approved by the Medical Ethics Committee in compliance with the local institutional review board regulations [protocol number: U2017.003]. Clinical parameters required for this study were retrospectively extracted from (electronic) patient records.

IHC analysis

Detailed information about the immunohistochemistry staining is provided in the Supplementary Material (Supplementary Material and Supplementary Table 1).

\section{Scoring of tumour PD-L1 expression}

Tumour PD-L1 scoring was performed according to the instruction manual of the qualitative immunohistochemical assay developed by Dako as a companion diagnostic tool for nivolumab [PharmDx] using the 28.8 antibody. Tumour PD-L1 expression levels were determined by observing complete circumferential or partial linear expression (at any intensity) of PD-L1 on the plasma cell membrane of viable tumour cells. In parallel, the pattern of staining in CD4-stained slides was evaluated and compared with PD-L1-stained slides in order to avoid false-positive assessment due to PD-L1-expressing macrophages. Assessment of expression levels was performed in sections that included at least 100 evaluable tumour cells. Heterogeneous PD-L1 staining was evaluated according to the instruction of the kit, by dividing the tumour areas into sections with equal amounts of tumour cells at a low magnification, and scoring the percentage of PD-L1-positive cells for each area separately. Next the percent positivity from each area was added and divided by the total number of areas. $^{16,17}$ The percentage of stained tumour cells in the entire specimen was categorised into the following categories: $<1 \%$, $1-5 \%, 5-10 \%, 10-25 \%, 25-50 \%$ and $\geq 50 \%$. Assessment of tumour PD-L1 expression, as well as of the other IHC parameters, was performed by a senior experienced thoracic pathologist (ET) and blinded for treatment outcome.

Scoring of immune cell infiltrates

Immune cell infiltration was assessed by evaluating the presence of PD- $\mathrm{L}^{+}{ }_{-}, \mathrm{PD}_{-} 1^{+}, \mathrm{CD}^{+}{ }_{-}, \mathrm{CD}^{+}{ }^{+}$and $\mathrm{CD} 8^{+}$-stained immune cells in stromal tissue and $\mathrm{CD}^{+}$immune cells in tumour tissue. For assessment of stroma only, the peritumoural stromal area was scored, because of its prognostic importance. ${ }^{14,18}$ The peritumoural stroma was defined as the stroma directly adjacent to tumour cell areas. The density of immune cell infiltrates in the stromal and tumour compartments was scored as no infiltration, low infiltration, intermediate infiltration and high infiltration according to Paulsen et al. and Donne et al. ${ }^{19,20}$ To ensure a more detailed categorisation, we added an extra category 'very low infiltration' to the existing scale with occasionally immune cells present. In the case of heterogeneous infiltration, the highest identifiable infiltration category was scored.

Data collection

Clinical data on patients' gender, age at the start of treatment, performance score and tumour histology were extracted from the available databases. Baseline CT scan of thorax $+/-$ abdomen had to be obtained within 6 weeks before the start of treatment. Response evaluation was performed using $C T$ imaging every 6 weeks or, after a partial response was obtained, every 3 months according to the institutional treatment protocol.

Treatment outcome

The primary outcome measure of this study was DCB defined as complete response $(C R)$, partial response $(P R)$ or stable disease (SD) by RECIST $v 1.1$ for a duration of $\geq 6$ months after the start of nivolumab treatment. ${ }^{21}$ All available CT scans were scored blinded for the IHC results. Patients who were diseased or deteriorated before the first response assessment, were designated as patients without DCB. According to these data, two treatment outcome groups, patients with $D C B$ and without $D C B$, were defined.

\section{Statistics}

Mean with standard deviation (SD) were used as descriptive statistics for data that were normally distributed. Median with interquartile range (IQR) were used for not normally distributed data. For differences between two unpaired groups, the chisquare test was used for dichotomous or ordinal variables, and the independent samples' $t$ test or Wilcoxon rank-sum test (when the data were not normally distributed) for continuous variables. In order to attain sufficiently large groups for analysis, selected categories of infiltration by immune cells were combined based on group size (see Supplementary Table 2).

The following categories were combined, based on previous studies: stromal $\mathrm{CD}^{+}$and tumour $\mathrm{CD}^{+}$immune cell infiltration, stromal PD $-1^{+}$and $\mathrm{CD}^{+}$immune cell infiltration and stromal PD$\mathrm{L}^{+}$and $\mathrm{CD}^{+}$immune cell infiltration. ${ }^{14,15,22,23}$

The final dataset was 1:1 randomised into a derivation cohort and a validation cohort at random by SPSS. The derivation cohort was used to examine the association between individual or combined IHC variables and DCB using univariate logistic regression models. The validation cohort was used to validate the observed associations between the study variables and DCB to nivolumab in a multivariate logistic regression model, built with a forward selection procedure ( $p$ entry $<0.1$ ). OS was analysed using the Kaplan-Meier method and compared between two unpaired groups using log-rank tests. Hazard ratios (HRs) were estimated with univariate Cox proportional-hazard models for the expression levels of tumour and stromal markers with corresponding $95 \%$ confidence interval $(\mathrm{Cl})$. Statistical analyses were performed using SPSS, version 22 (IBM Corp., Armonk, NY). Figures were generated with GraphPad Prism, version 7.02. $P$ values $<0.05$ were considered to indicate statistical significance.

\section{RESULTS}

In total, 194 patients were treated with nivolumab in the period of August 2015 till January 2017. Fifty-five patients were excluded because (1) no suitable biopsy samples were obtained prior to treatment $(n=36)$; (2) available biopsy samples did not contain sufficient tumour tissue $(n=17)$; (3) because patients were on combined therapy (tyrosine kinase inhibitors and immunotherapy) at the start of the treatment $(n=2)$ (Fig. 1a). Hundred-thirty-nine 


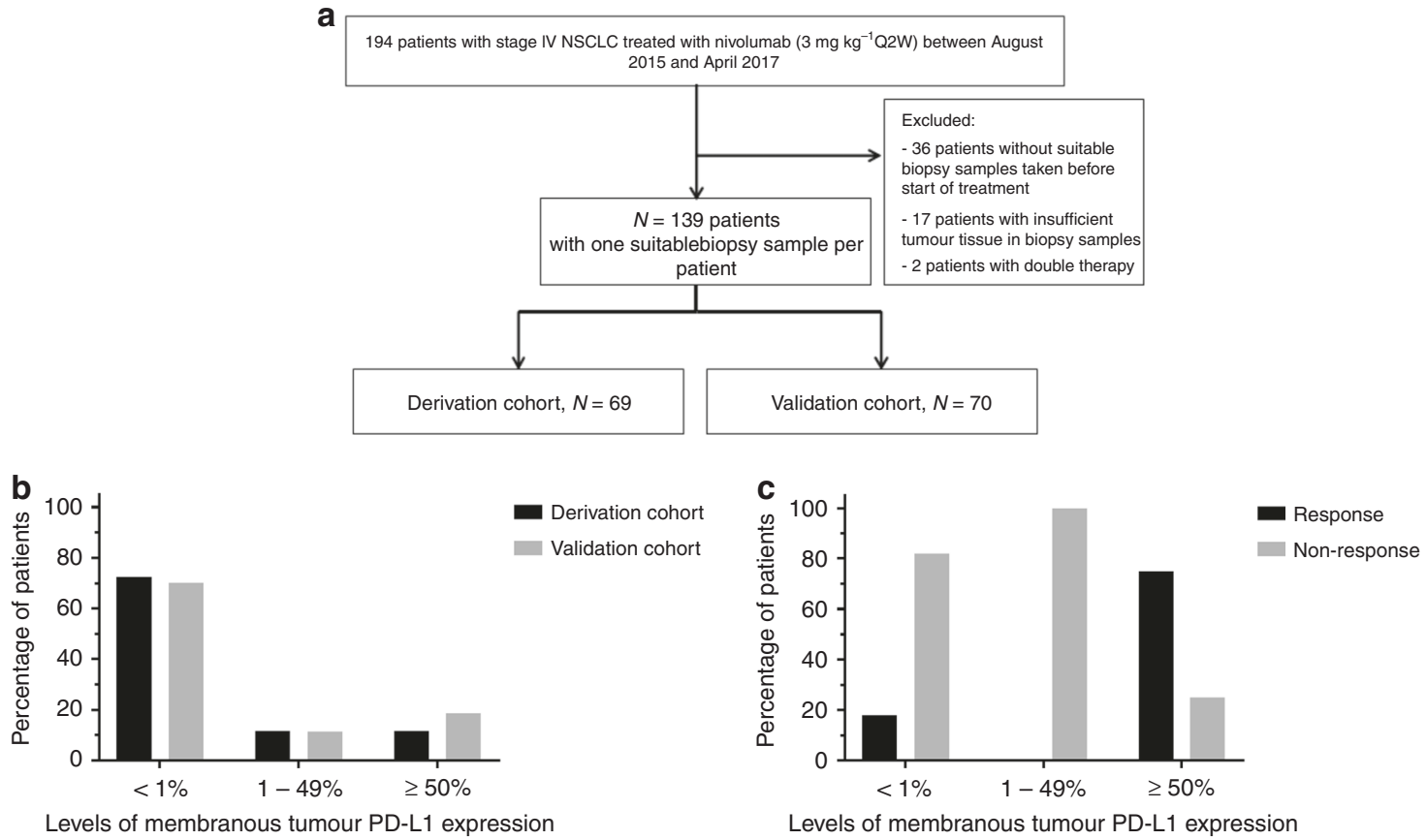

Fig. 1 Flowchart of the study and distribution of PD-L1 and relation with durable clinical benefit (DCB). a Flowchart demonstrating the selection procedure of patient biopsy samples. b Distribution of tumour PD-L1 expression in both cohorts. In both cohorts, the largest proportion of patients had $<1 \%$ tumour PD-L1 expression. c Tumour PD-L1 expression was associated with DCB in the derivation cohort.

Table 1. Baseline characteristics.

\begin{tabular}{|c|c|c|c|}
\hline & $\begin{array}{l}\text { Derivation, } \\
n(\%)\end{array}$ & $\begin{array}{l}\text { Validation, } \\
n(\%)\end{array}$ & $p$ value \\
\hline Number of patients & 69 & 70 & \\
\hline Mean age in years, mean \pm SD & $62.3 \pm 10.7$ & $63.7 \pm 7.2$ & 0.37 \\
\hline Number of biopsy samples & 69 & 70 & 0.87 \\
\hline $\begin{array}{l}\text { Obtained after the last line of } \\
\text { treatment }(\mathrm{ACT})\end{array}$ & $36(52.2)$ & $35(50.0)$ & \\
\hline $\begin{array}{l}\text { Obtained before the last line } \\
\text { of treatment }(B C T)\end{array}$ & $33(47.8)$ & $35(50.0)$ & \\
\hline Sex & & & 0.61 \\
\hline Male & $40(58.0)$ & $37(52.9)$ & \\
\hline Female & $29(42.0)$ & $33(47.1)$ & \\
\hline Histological subtypes & & & 0.31 \\
\hline Squamous & $20(29.0)$ & $21(68.6)$ & \\
\hline Nonsquamous & $46(66.7)$ & $48(30.0)$ & \\
\hline $\begin{array}{l}\text { Other (adenosquamous, } \\
\text { LCNEC, NOS or unknown) }\end{array}$ & $3(4.3)$ & $1(1.4)$ & \\
\hline ECOG performance score & & & 0.59 \\
\hline 0 & $7(10.1)$ & $8(11.4)$ & \\
\hline 1 & $44(63.8)$ & $40(57.1)$ & \\
\hline$\geq 2$ & $16(23.2)$ & $22(31.4)$ & \\
\hline Unknown & $2(2.9)$ & $0(0)$ & \\
\hline$D C B$ rate & & & 0.34 \\
\hline Patients without DCB & $15(21.7)$ & $21(30.0)$ & \\
\hline Patients with DCB & $54(78.3)$ & $50(70.0)$ & \\
\hline
\end{tabular}

biopsy samples from 139 patients with advanced-stage NSCLC were included in this study. Patient and tumour characteristics for the derivation and validation cohorts are shown in Table 1. None of the baseline characteristics differed significantly among the cohorts. All patients received nivolumab as second-line treatment or beyond, after prior treatment with platinum-doublet chemotherapy + /- local treatment with radiotherapy or surgery. In the derivation cohort, $21.7 \%$ of patients showed DCB to nivolumab, versus $30.0 \%$ of patients in the validation cohort.

Tumour PD-L1 expression and stromal and tumour immune cell infiltration are associated with DCB and OS in the derivation cohort

The distribution of the levels of membranous PD-L1 expression in the derivation and the validation cohorts is visualised in Fig. $1 \mathrm{~b}$ and described in Supplementary Table 3. The fraction of patients with $<1 \%$ membranous PD-L1 expression in tumour tissue in the derivation and validation cohorts was $72.5 \%$ and $70.0 \%$, respectively. Likewise, the fraction of patients with $\geq 50 \%$ membranous PD-L1 expression in the validation and derivation cohorts was $11.6 \%$ and $18.6 \%$, respectively. For the category with membranous PD-L1 expression in tumour tissue between 1 and 49\%, the fraction of patients in the validation and derivation cohorts was $14.3 \%$ and $11.4 \%$, respectively $(p=0.62)$.

The results of univariate and multivariate analyses performed on the derivation cohort are shown in Table 2. In univariate analysis, tumour PD-L1 was associated with DCB, especially in the patients with $\geq 50 \%$ tumour PD-L1 expression (DCB rate $75 \%$, OR 13.6, 95\% Cl 2.3-79.0) (Fig. 1C and Table 2). Multivariate analysis confirmed this association. This finding was subsequently evaluated in the validation cohort. The DCB rate was 39\% in the validation cohort for tumour PD-L1 $\geq 50 \%$, and tumour PD-L1 expression was not associated with $D C B$ in the validation cohort $(p=0.63)$, see Supplementary Table 4 .

The levels of PD- $1^{+}, \mathrm{PD}-1^{+}, \mathrm{CD}^{+}, \mathrm{CD}^{+}$and $\mathrm{CD} 4^{+}$immune cell infiltration in tumour and/or stroma in samples of both cohorts are presented in Supplementary Table 3. No significant differences were observed between both groups. Univariate analysis revealed a significant association between stromal PD$1^{+}$immune cell infiltration, stromal $\mathrm{CD}^{+}$immune cell infiltration, stromal $\mathrm{CD}^{+}$immune cell infiltration, combined tumour and stromal infiltration of $\mathrm{CD}^{+}$immune cells, combined intermediateto-high stromal infiltration of $\mathrm{CD}^{+}$and PD- $1^{+}$immune cells and 
Association of tumour and stroma PD-1, PD-L1, CD3, CD4 and CD8 expression... A.-L.N Niemeijer et al.

Table 2. Results of univariate analysis of PD-L1, PD-1, CD8, CD4 and CD3 in relation to durable clinical benefit are shown for the derivation cohort.

Variables

Derivation cohort

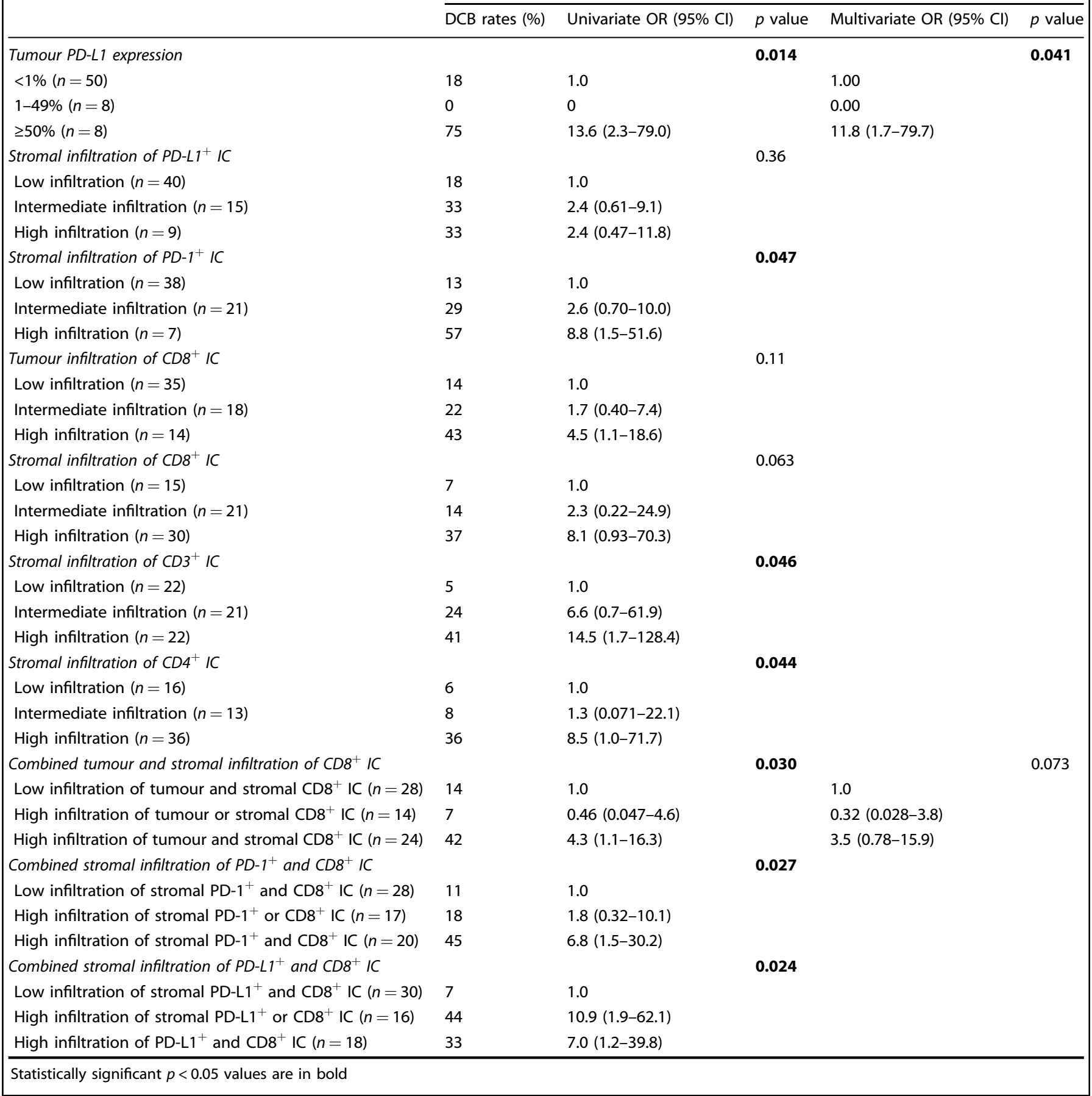

combined stromal infiltration by $\mathrm{PD}-\mathrm{L}^{+}$and $\mathrm{CD} 8^{+}$immune cells and DCB (Table 2). However, multivariate analysis could not confirm these associations (Table 2), and the univariate associations were not confirmed by univariate analysis in the validation cohort (Supplementary Table 4).

Overall survival in all categories is shown in Table 3 for the derivation and the validation cohorts. In the derivation cohort, high tumour PD-L1 expression $(p=0.003)$, high infiltration of stromal $\mathrm{CD}^{+}$immune cells $(p=0.035)$, high infiltration of stromal $\mathrm{CD}^{+}$immune cells $(p=0.017)$, high infiltration of stromal $\mathrm{CD}^{+}$immune cells $(p=0.010)$, both high infiltration of stromal and tumour $\mathrm{CD}^{+}$immune cells $(p<0.0001)$, both high infiltration of stromal PD- $1^{+}$and $\mathrm{CD}^{+}$immune cells $(p=0.023)$ and both high infiltration of stromal PD-L1 ${ }^{+}$and $\mathrm{CD} 8^{+}$immune cells $(p=0.021)$ were associated with longer OS (Fig. $2 a-g$, Table 3).

In the validation cohort, longer OS was observed for patients with high infiltration of $\mathrm{CD}^{+}$immune cells, $(p=0.024)$, patients 
Table 3. Overall survival of PD-L1, PD-1, CD8, CD4 and CD3 is shown for the derivation and validation cohorts.

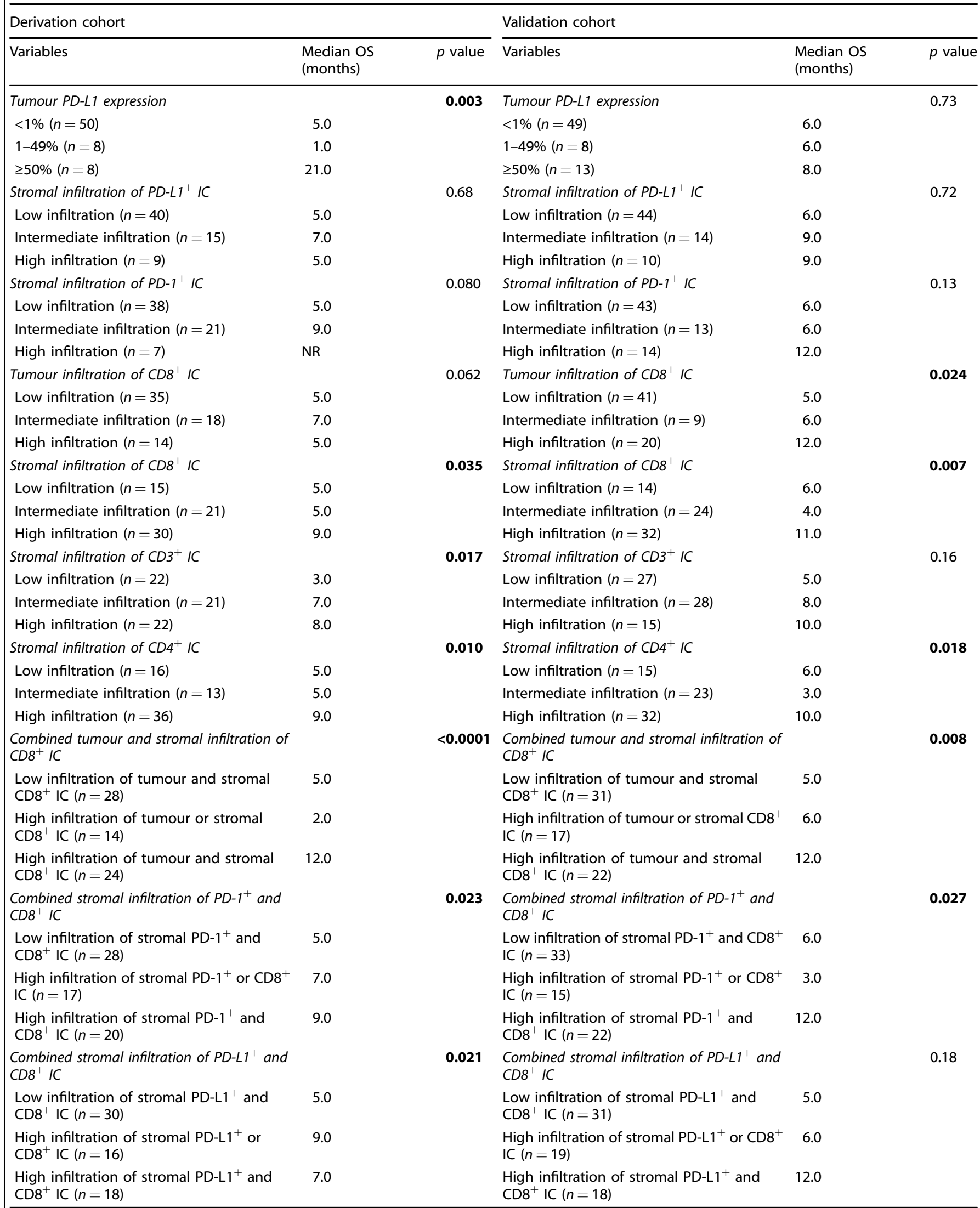

Statistically significant $p<0.05$ values are in bold 

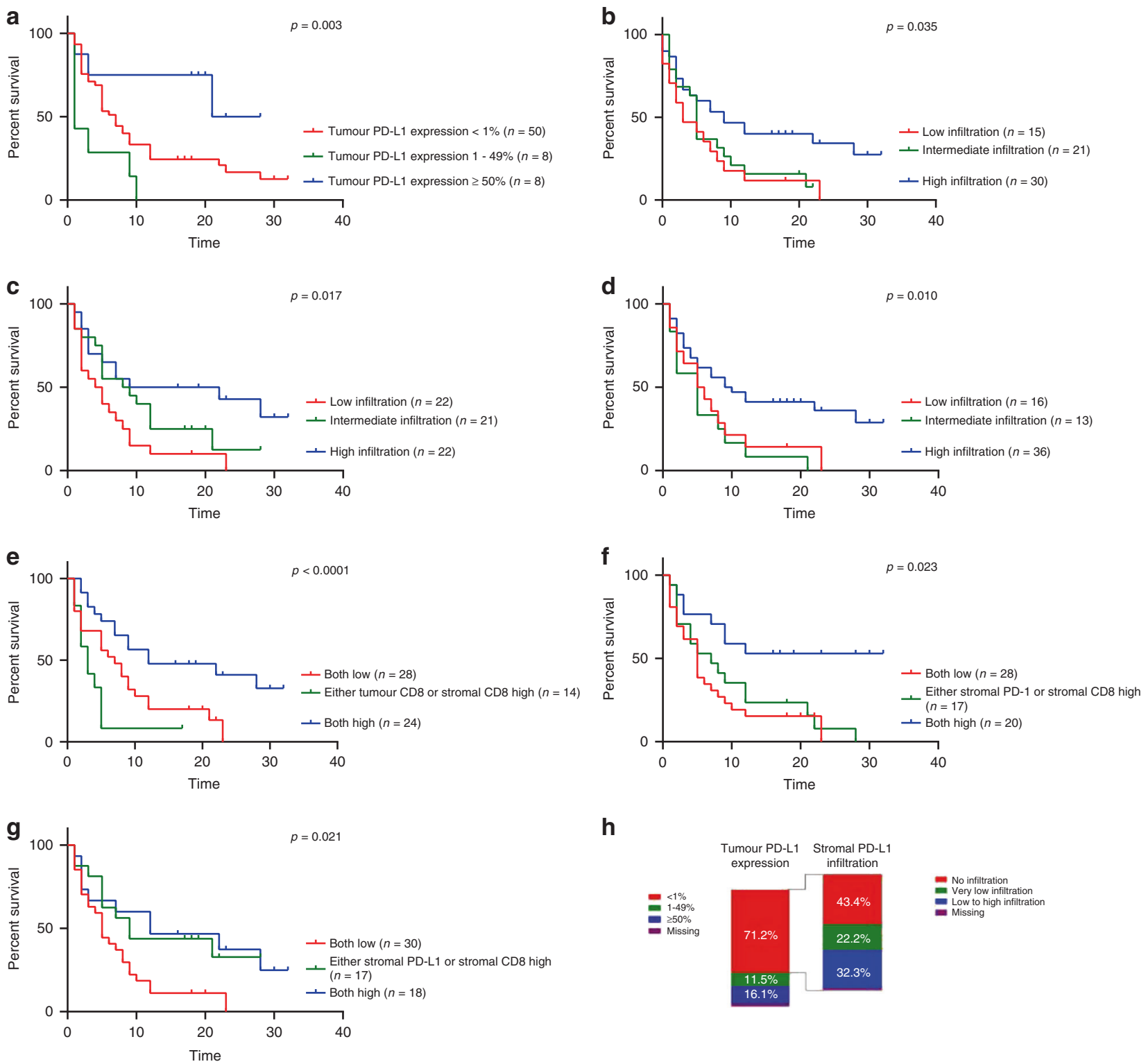

h

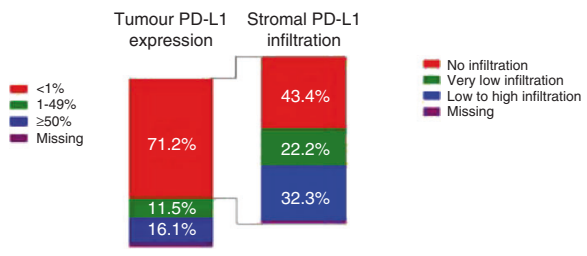

Fig. 2 Kaplan-Meier plots for the derivation cohort. a Survival of tumour PD-L1 expression in the derivation cohort. b Survival of stromal $\mathrm{CD}^{+}$infiltration in the derivation cohort. c Survival of stromal $\mathrm{CD}^{+}$infiltration in the derivation cohort. d Survival of stromal CD4 ${ }^{+}$infiltration in the derivation cohort. e Survival of combined tumour and stromal CD8 ${ }^{+}$infiltration in the derivation cohort. $f$ Survival of stromal combined PD- $1^{+}$and $C D 8^{+}$infiltration in the derivation cohort. g Survival of stromal combined PD-L1 ${ }^{+}$and $\mathrm{CD} 8^{+}$infiltration in the derivation cohort. h Distribution of stromal PD-L1 expression in tumour PD-L1-negative patients (tumour PD-L1 expression < 1\%).

with high expression of stromal $\mathrm{CD}^{+}$immune cells $(p=0.007)$, patients with high infiltration of stromal and tumour-infiltrating $\mathrm{CD}^{+}$immune cells $(p=0.008)$ and patients with high stromal infiltration of both PD- $1^{+}$and $\mathrm{CD}^{+}$immune cells $(p=0.027$, Supplementary Fig. 1; Table 3).

In the total cohort, combined tumour and stromal $\mathrm{CD}^{+}$immune cell infiltration is associated with DCB in multivariate analysis, and immune cell markers are associated with OS

As we were not able to confirm our results of the derivation cohort in the validation cohort for DCB, we decided to combine both cohorts to enlarge the cohort size. In the total cohort, approximately $15 \%$ of all patients had positive membranous tumour PD-L1 staining of $\geq 50 \%$, and $71.2 \%$ of all patients had less than $1 \%$ membranous tumour PD-L1 staining (Supplementary Table 5; Fig. 2h). In these tumour PD-L1-negative tumours, more than $50 \%$ of the patients had stromal infiltration of PD-L1-positive immune cells (Fig. 2h).

In the total cohort, univariate analyses showed that, besides the previously described tumour PD-L1 expression, also stromal CD3 ${ }^{+}$, stromal $\mathrm{CD}^{+}{ }^{+}$, combined stromal infiltration of $\mathrm{CD}^{+}$and $\mathrm{PD}-1^{+}$ immune cells and tumour and stromal infiltration of $\mathrm{CD}^{+}$ immune cells were associated with DCB (Supplementary Table 6). Multivariate analysis showed that infiltration of combined stromal and tumour $\mathrm{CD}^{+}$immune cells was associated with $\mathrm{DCB}$ (Supplementary Table 6).

Median OS was significantly longer for patients with high stromal PD- $1^{+}$immune cell infiltration $(p=0.015)$, patients with high tumour $\mathrm{CD}^{+}$immune cell infiltration $(p=0.004)$, patients with high stromal $\mathrm{CD}^{+}$immune cell infiltration $(p=0.001)$, patients with high infiltration of stromal $\mathrm{CD}^{+}$immune cells $(p=0.004)$, patients with high stromal $\mathrm{CD}^{+}$immune cell infiltration $(p<0.0001)$, patients 
with high infiltration of both tumour and stromal $C D 8^{+}$immune cell infiltration $(p<0.0001)$, patients with high stromal infiltration of both PD- $1^{+}$and $\mathrm{CD} 8^{+}$immune cells $(p=0.001)$ and patients with high infiltration of both stromal PD-L1 ${ }^{+}$and $\mathrm{CD}^{+}$immune cells $(p=$ 0.013) (Supplementary Fig. 2a-h, Supplementary Table 7).
Tumour PD-L1 expression and immune cell infiltration markers predict DCB when analysed on tumour tissue taken after the last line of therapy, but not on archival samples

To address the influence of tumour biopsy timing (before or after the last line of systemic treatment), we performed a post hoc

Table 4. Results of univariate analysis of PD-L1, PD-1, CD8, CD4 and CD3 in relation to durable clinical benefit (DCB) are shown for biopsies taken before chemotherapy $(B C T)$ and biopsies taken after the last line of chemotherapy (ACT).

BCT biopsies

ACT biopsies

DCB rates (\%) Univariate $\mathrm{OR} \quad p$ value $(95 \% \mathrm{Cl})$

Tumour PD-L1 expression

$<1 \%(n=52)$

$1-49 \%(n=7)$

$\geq 50 \%(n=8)$

Stromal infiltration of $P D-L 1^{+} I C$

Low infiltration $(n=39) \quad 26$

Intermediate infiltration $(n=15) \quad 13$

High infiltration $(n=11) \quad 18$

Stromal infiltration of $P D-1^{+} I C$

Low infiltration $(n=40) \quad 25$

Intermediate infiltration $(n=17) \quad 12$

High infiltration $(n=9) \quad 22$

Tumour infiltration of $\mathrm{CD} 8^{+} / C$

Low infiltration $(n=37) \quad 22$

Intermediate infiltration $(n=11) 9$

High infiltration $(n=18) \quad 28$

Stromal infiltration of $\mathrm{CD}^{+}$IC

Low infiltration $(n=11) \quad 18$

Intermediate infiltration $(n=28) 18$

High infiltration $(n=27) \quad 26$

Stromal infiltration of $\mathrm{CD}^{+} \mathrm{IC}$

Low infiltration $(n=23) \quad 9$

Intermediate infiltration $(n=26) \quad 31$

High infiltration $(n=17)$

Stromal infiltration of $\mathrm{CD}^{+} \mathrm{IC}$

Low infiltration $(n=16) \quad 19$

Intermediate infiltration $(n=19) 11$

High infiltration $(n=31) \quad 29$

Combined tumour and stromal

infiltration of $\mathrm{CD}^{+}$IC

Low infiltration of tumour and 21

stromal $\mathrm{CD}^{+} \mathrm{IC}(n=28)$

High infiltration of tumour or 15

stromal $\mathrm{CD}^{+}$IC $(n=20)$

High infiltration of tumour and 28

stromal $\mathrm{CD}^{+}$IC $(n=18)$

Combined stromal infiltration of

$P D-1^{+}$and $C D 8^{+}$IC

Low infiltration of stromal 19

PD- $1^{+}$and $\mathrm{CD}^{+}$IC $(n=31)$

High infiltration of stromal PD- 29

$1^{+}$or $\mathrm{CD}^{+}$IC $(n=17)$

High infiltration of stromal PD- 17

$1^{+}$and $\mathrm{CD}^{+}$IC $(n=18)$

Combined stromal infiltration of

$P D-L 1^{+}$and $C D 8^{+}$IC

Low infiltration of stromal 19

$\mathrm{PD}-\mathrm{L} 1^{+}$and $\mathrm{CD} 8^{+} \mathrm{IC}(n=31)$

High infiltration of stromal PD- 33

$\mathrm{L}^{+}$or $\mathrm{CD}^{+} \mathrm{IC}(n=15)$

High infiltration of stromal PD- 16

$\mathrm{L}^{+}$and $\mathrm{CD}^{+} \mathrm{IC}(n=19)$

(95\% CI)

1

$0.62(0.068-5.7)$

$1.2(0.22-7.0)$

1

$0.45(0.085-2.3)$

$0.64(0.12-3.5)$

1

$0.40(0.078-2.1)$

$0.86(0.15-4.8)$

1

$0.36(0.040-3.3)$

$1.4(0.38-5.1)$

1

$0.98(0.16-6.0)$

$1.6(0.27-9.1)$

1

$4.7(0.88-24.9)$

$3.2(0.52-20.2)$

1

$0.51(0.074-3.5)$

$1.8(0.41-7.8)$

1

$0.65(0.14-3.0)$

$1.4(0.36-5.6)$

1

$1.7(0.44-6.8)$

$0.83(0.18-3.8)$

1

$2.1(0.52-8.4)$

$0.78(0.17-3.6)$

0.88 Tumour PD-L1 expression

$<1 \%(n=47)$

$1-49 \%(n=9)$

$\geq 50 \%(n=13)$

0.60

Stromal infiltration of PD-L1 ${ }^{+}$IC

Low infiltration $(n=45)$

Intermediate infiltration $(n=14)$

High infiltration $(n=8) \quad 50$

0.55 Stromal infiltration of PD- ${ }^{+}$IC

Low infiltration $(n=41) \quad 17$

Intermediate infiltration $(n=17) 41$

High infiltration $(n=12) \quad 67$

0.52 Tumour infiltration of $\mathrm{CD}^{+} \mathrm{IC}$

Low infiltration $(n=39) \quad 18$

Intermediate infiltration $(n=16) \quad 31$

High infiltration $(n=16) \quad 63$

0.74 Stromal infiltration of $\mathrm{CD}^{+} \mathrm{IC}$

Low infiltration $(n=18) \quad 17$

Intermediate infiltration $(n=17) \quad 18$

High infiltration $(n=35) \quad 46$

0.20 Stromal infiltration of $\mathrm{CD}^{+} \mathrm{IC}$

Low infiltration $(n=26) \quad 19$

Intermediate infiltration $(n=23) 26$

High infiltration $(n=20)$

0.31 Stromal infiltration of $\mathrm{CD}^{+} \mathrm{IC}$

Low infiltration $(n=15) \quad 20$

Intermediate infiltration $(n=17) \quad 18$

High infiltration $(n=37)$

0.64 Combined tumour and stromal infiltration of $\mathrm{CD}^{+} \mathrm{IC}$

Low infiltration of tumour and stromal $\mathrm{CD}^{+} \mathrm{IC}(n=31)$

High infiltration of tumour or stromal $\mathrm{CD}^{+} \mathrm{IC}(n=11)$

High infiltration of tumour and 54 stromal CD8 ${ }^{+}$IC $(n=28)$

62 Combined stromal infiltration of PD- $1^{+}$and $\mathrm{CD}^{+} \mathrm{IC}$

Low infiltration of stromal PD-1 ${ }^{+} 20$ and $\mathrm{CD}^{+} \mathrm{IC}(n=30)$

High infiltration of stromal $\mathrm{PD}^{-1}{ }^{+}$or $\mathrm{CD8}{ }^{+} \mathrm{IC}(n=15)$

High infiltration of stromal

PD- $1^{+}$and $\mathrm{CD}^{+} \mathrm{IC}(n=24)$

0.44 Combined stromal infiltration of $P D-L 1^{+}$and $C D 8^{+}$IC

Low infiltration of stromal

$\mathrm{PD}-\mathrm{L}^{+}$and CD8 ${ }^{+}$IC $(n=30)$

High infiltration of stromal

$\mathrm{PD}-\mathrm{L} 1^{+}$or $\mathrm{CD} 8{ }^{+}$IC $(n=20)$

High infiltration of stromal $\mathrm{PD}-\mathrm{L}^{+}$and $\mathrm{CD}^{+} \mathrm{IC}(n=17)$
DCB rates (\%) Univariate OR (95\% Cl)

$(95 \%$ CI)

$0.94(0.17-5.2)$

$7.4(1.9-28.6)$

1

$2.3(0.66-8.2)$

$3.1(0.66-14.5)$

$3.4(0.96-12.0)$

$9.7(2.3-41.4)$

$2.1(0.55-7.9)$

$7.6(2.1-28.0)$

$1.1(0.19-6.2)$

$4.2(1.0-17.2)$

0.035

1

1,5 (0.39-5.7)

5.1 (1.4-19.1)

1

$0.86(0.15-5.1)$

$3.0(0.74-12.6)$

0.008

1

$0.42(0.044-3.9)$

$4.8(1.5-15-3)$

0.001

1

$0.29(0.031-2.6)$

$6.7(2.0-22.5)$

0.043

Statistically significant $p<0.05$ values are in bold 
Table 5. Multivariate analysis for biopsies taken after the last line of therapy (ACT).

\begin{tabular}{lll}
\hline & DCB rates (\%) & Multivariate OR (95\% CI) \\
\hline Tumour PD-L1 expression & 23 & 1.0 \\
$<1 \%(n=47)$ & 22 & $0.047(0.13-4.8)$ \\
$1-50 \%(n=9)$ & 69 & $6.5(1.4-30.7)$ \\
$\geq 50 \%(n=13)$ & & 1.0 \\
Combined tumour and stromal infiltration of CD $8^{+} I C$ & 19 & $0.29(0.026-3.1)$ \\
Low infiltration of tumour and stromal CD8 & \\
High infiltration of tumour or stromal $C D 8^{+} \mathrm{IC}(n=31)$ & 9 & $4.5(1.3-15.9)$ \\
High infiltration of tumour and stromal CD8 $8^{+} \mathrm{IC}(n=28)$ & 54 & $\mathbf{0 . 0 1 2}$ \\
\hline Statistically significant $p<0.05$ values are in bold & & \\
\hline
\end{tabular}

analysis between those patient groups. Baseline characteristics were equal with the exception of ECOG performance score ( $p=$ 0.002) (Supplementary Table 8). Infiltration levels of immune cells were equal (Supplementary Table 9). In the ACT biopsy group, tumour PD-L1 expression associated with DCB rate (DCB rate $69 \%$ for PD-L1 $\geq 50 \%$, OR 7.4, 95\% Cl 1.9-28.6), but not in the BCT biopsy group (Table 4). In univariate analyses, stromal PD-1 ${ }^{+}$ immune cell infiltration, tumour $\mathrm{CD}^{+}$immune cell infiltration, stromal $\mathrm{CD}^{+}$immune cell infiltration, stromal $\mathrm{CD}^{+}{ }^{+}$immune cell infiltration, combined tumour and stromal $\mathrm{CD}^{+}$immune cell infiltration, combined stromal infiltration of $\mathrm{PD}^{-} 1^{+}$and $\mathrm{CD}^{+}$ immune cells and combined stromal PD- $1^{+}$and $\mathrm{CD}^{+}$immune cell infiltration were associated with DCB in the ACT biopsy group. In the BCT biopsy group, no significant associations were found. Multivariate analysis showed that tumour PD-L1 and combined tumour and stromal infiltration of $\mathrm{CD}^{+}$immune cells were associated with DCB in the ACT biopsy group (Table 5).

In the ACT biopsy group, we found that high infiltration of stromal PD- $1^{+}$immune cell infiltration $(p=0.005)$, high infiltration of tumour $\mathrm{CD}^{+}$immune cells $(p=0.019)$, high infiltration of stromal $\mathrm{CD}^{+}$immune cells $(p=0.001)$, high infiltration of $\mathrm{CD}^{+}$ immune cells $(p=0.003)$, high infiltration of $\mathrm{CD}^{+}$immune cells $(p=0.001)$, both high infiltration of tumour and stromal $\mathrm{CD}^{+}$ immune cells $(p<0.0001)$, high infiltration of both PD $-1^{+}$and $\mathrm{CD}^{+}$immune cells $(p<0.0001)$ and both high infiltration of stromal PD-L1 ${ }^{+}$and $\mathrm{CD}^{+}$immune cells $(p=0.002)$ were associated with longer OS (Supplementary Fig. 3a-h, Table 6). In the BCT biopsy group, we found no associations between OS and immune cell infiltration.

\section{DISCUSSION}

In this explorative study, we found that tumour PD-L1 expression was predictive for DCB in the derivation cohort, and in patients who have had a biopsy after the last line of therapy. Although only confirmed in the latter group and in the total cohort, the infiltration of both tumour and stromal $\mathrm{CD}^{+}$immune cells seems to play an additional role in the predictive value for DCB. High infiltration of stromal $\mathrm{CD}^{+}$immune cells, high infiltration of stromal $\mathrm{CD}^{+}{ }^{+}$immune cells, high infiltration of both tumour and stromal $\mathrm{CD}^{+}$immune cells and high infiltration of PD-1 ${ }^{+}$ and $\mathrm{CD}^{+}$immune cells were associated with longer OS in all subsets. This implies that the microenvironment of the tumour and the stromal compartment is important for obtaining a durable response to nivolumab.

Consistent with earlier studies in melanoma and NSCLC patients, tumour and stromal infiltration of $\mathrm{CD}^{+}{ }^{+}$immune cells were associated with DCB. ${ }^{14,22,23}$ In line with these results, we also observed stromal $\mathrm{CD}^{+}$infiltration alone to be associated with OS. In contrast to a small study among NSCLC patients treated with nivolumab, that showed that low PD-1 to CD8 ratio was associated with $D C B$ and progression-free survival (PFS), we found that high stromal infiltration of both PD- $1^{+}$and $\mathrm{CD}^{+}$immune cells was associated with DCB and OS. Similar findings were reported in previous studies in melanoma patients. ${ }^{14,24}$ The upregulation of PD-1 on $\mathrm{CD}^{+} \mathrm{T}$ cells under chronic and persistent antigen stimulation is well described. ${ }^{2}$ Blocking this PD-1 receptor with anti-PD-1 therapy could therefore result in activation of $\mathrm{CD}^{+}$ $T$ cells, and as a result enhance tumour regression.

The role of $\mathrm{CD}^{+}{ }^{+} \mathrm{T}$ cells is increasingly being studied in the $\mathrm{T}$-cell response against tumours. $\mathrm{CD}^{+}{ }^{+}$cells are responsible for cytokine production and T-cell regulation. A previous metaanalysis described that stromal infiltration of $\mathrm{CD}^{+}{ }^{+} \mathrm{T}$ cells is associated with better OS and disease-specific survival. ${ }^{25}$ In our study, the presence of stromal $\mathrm{CD}^{+} \mathrm{T}$ cells was also associated with a longer OS to nivolumab in the second-line setting.

Importantly, this study showed differences for the predictive value of IHC markers between biopsies obtained before and after the line of systemic treatment. While tumour PD-L1 and combined tumour and stromal infiltration of $\mathrm{CD}^{+}$immune cells were associated with $D C B$ and OS in the ACT biopsy group, there were no predictive markers for DCB and OS in the BCT biopsy group. Increasing evidence supports the hypothesis that the anticancer effects of chemotherapy not only cause cancer apoptosis, but also modulate the immune system. The widely used drug cisplatin, for example, induces upregulation of MHC class I expression on tumour cells and antigen-presenting cells, improves the proliferation of immune effector cells and can downregulate immunosuppressive components in the tumour microenvironment. ${ }^{26}$ Changes in PD-L1 expression and immune contexture during anticancer treatment have been reported. ${ }^{27-30}$ OS benefit of pembrolizumab over docetaxel has been demonstrated for both $\geq 50 \%$ and $\geq 1 \%$ tumour PD-L1 expression cohorts, regardless of the time the samples were collected, ${ }^{31}$ whereas in this study, no association between tumour PD-L1 expression and stromal infiltrates and response was found in the BCT biopsy group. Our results suggest that obtaining a new biopsy before the start of treatment is warranted in the case the patient received chemotherapy.

Recently, an increasing number of studies are indicating that tumour-infiltrating immune cells like dendritic cells and macrophages contribute to the antitumour effect of anti-PD-(L)1 therapy. ${ }^{32,33}$ In this study, we observed that patients with high stromal infiltration of PD- $1^{+}$and $\mathrm{CD}^{+}$immune cells had a better OS than patients with low infiltration, although we could not confirm these results in our validation cohort. We did not selectively stain for dendritic cells or macrophages, but our results suggest that stromal expression of PD-L1 prolongs OS.

The use of PD-L1 expression as a biomarker for immune checkpoint inhibitors has been debated. In previous trials with nivolumab, the association between PD-L1 IHC and outcome parameters in terms of the overall response rate (ORR), OS and PFS has been conflicting. ${ }^{4,53}$ In our study, we found that tumour 
Table 6. Overall survival of PD-L1, PD-1, CD8, CD4 and CD3 is shown for biopsies taken before chemotherapy (BCT) and biopsies taken after the last line of chemotherapy (ACT).

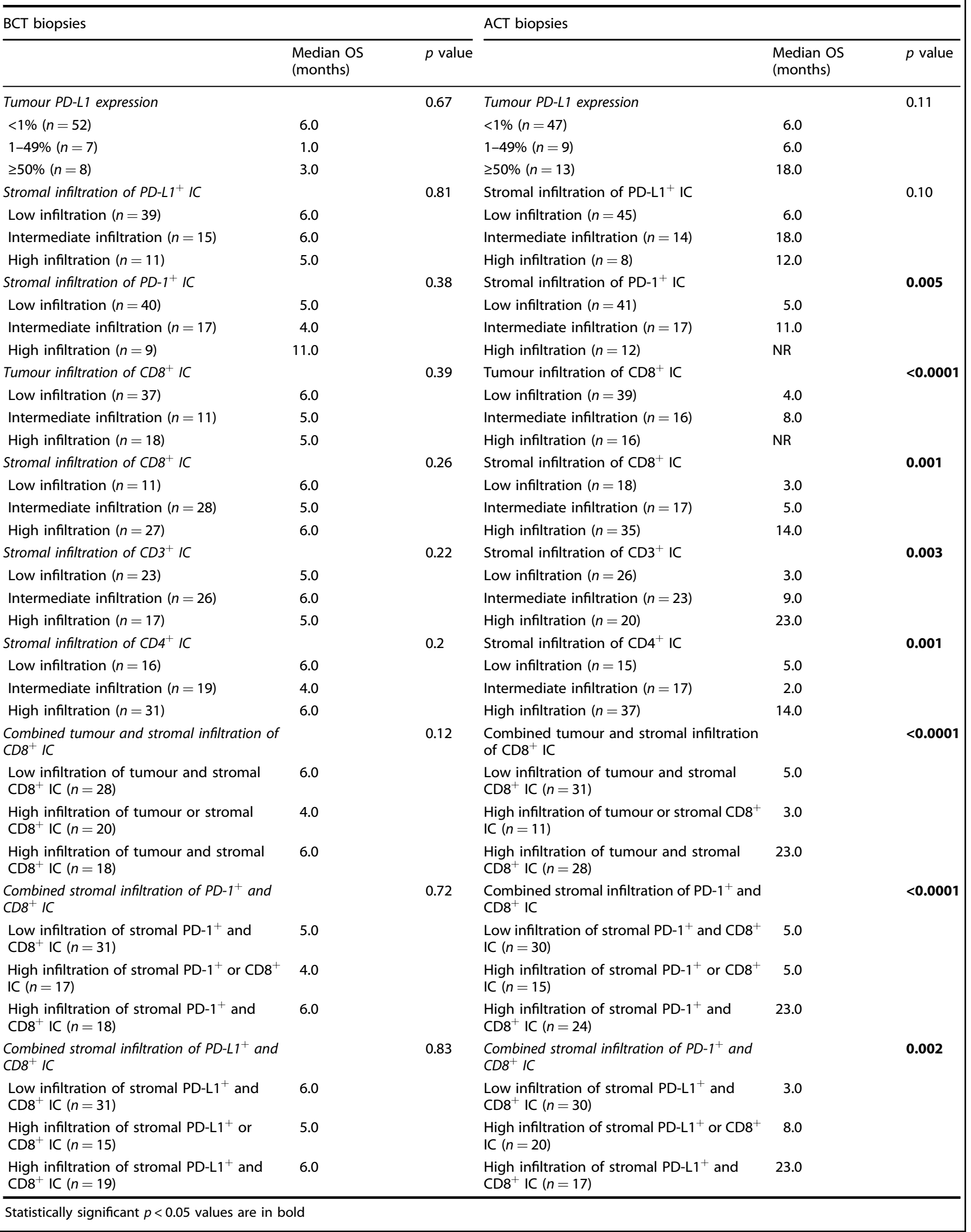


PD-L1 expression was associated with DCB, and with OS in only the derivation cohort. We also did not observe an association between stromal PD-L1 expression as a single marker and DCB. A previous study in patients treated with atezolizumab described durable responses in patients with PD-L1 positivity on immune cells alone. ${ }^{35}$ An explanation for this difference could be the difference in PD-L1 assays that were used to stain PD-L1, as this may explain misclassification of PD-L1 expression status. ${ }^{11}$

In this study, $71 \%$ of all patients had a negative tumour PD-L1 expression score. This contrasts with previous studies that reported prevalences between $17 \%$ and $22 \% .{ }^{4,5,9}$ One possible explanation for this finding is the method used for quantifying tumour PD-L1 expression in this study. We used a broad scale of immune cell markers in addition to PD-L1 for staining biopsy samples, which resulted in a more comprehensive evaluation of the different cell types that expressed PD-L1. When visual assessment of the nuclei did not yield a clear distinction between malignant and immune cells, the use of dual CD4 and PD-L1 staining helped to make the distinction between malignant and immune cells, thereby preventing overestimation of PD-L1 scores. It cannot be excluded that macrophages expressing both PD-L1 and CD4 were counted as PD-L1expressing tumour cells in a number of cases in previous studies, which may explain the difference between our observations and those reported in the literature, especially in the range of PD-L1 scoring below or just above the $1 \%$ range. Therefore, for future studies, simultaneous (multiplex) staining for PD-L1 and CD4 could result in improved accuracy of tumour PD-L1 assessments in biopsy samples.

There are several challenging aspects in the interpretation of our results. First, the heterogeneity and variability of PD-L1 expression within individual tumours may account for a limited external validity of our study, knowing that up to $35 \%$ of patients may be misclassified based on PD-L1 staining performed on small biopsy samples. ${ }^{36-39}$ Second, it is known that tumour PD-L1 expression levels can vary over time and be influenced by several host and environmental factors, such as TNM stage, chemotherapy and cytokines like interferon-a. ${ }^{40-43}$ In our study, both ACT and $B C T$ biopsies were included. Post hoc analysis shows that ACT biopsies were more informative for response prediction than $\mathrm{BCT}$ biopsies. Randomisation of our cohort in a derivation and validation cohort could not confirm our results found in the total cohort, with the exception of the association of immune cell markers and OS. This might be explained by the small numbers of patients in both cohorts.

In summary, in this study, we observed that tumour PD-L1, stromal infiltration of $\mathrm{CD}^{+}$immune cells, combined tumour and stromal infiltration of $\mathrm{CD}^{+}$immune cells, combined stromal infiltration of $\mathrm{PD}-1^{+}$and $\mathrm{CD}^{+}$immune cells and combined stromal infiltration of $\mathrm{PD}-\mathrm{L}^{+}$and $\mathrm{CD}^{+}{ }^{+}$immune cells are associated with $\mathrm{DCB}$ on nivolumab treatment. Although there were no major differences in baseline patient characteristics and the outcome to nivolumab treatment between the ACT and the $B C T$ biopsy groups, we were able to identify several predictive markers in the ACT group, including tumour PD-L1 expression and combined tumour and stromal infiltration of $\mathrm{CD} 8^{+}$immune cells for $\mathrm{DCB}$, and stromal PD- $1^{+}, \mathrm{CD}^{+}, \mathrm{CD}^{+}$and $\mathrm{CD}^{+}$ immune cells and tumour $\mathrm{CD}^{+}$immune cells for OS. The same markers in BCT biopsies were not predictive for DCB. This indicates that the immune contexture may change during treatment with conventional chemotherapy and therefore, that obtaining a new biopsy before the start of nivolumab is preferable. Further research is needed to validate our findings in a larger cohort, and to examine whether the infiltration of both tumour and stromal $\mathrm{CD}^{+}$immune cells can be used as a biomarker during immune checkpoint inhibition in NSCLC patients.

\section{AUTHOR CONTRIBUTIONS}

A.N., S.S., E.T. and A.J.L. were involved in the study design and conduct of this study. E.T. performed the scoring of IHC parameters. A.N., A.J.L. and E.F.S. performed the CT evaluation. A.N., S.S. and B.I.L.-W. performed statistical analyses. All authors were involved in the interpretation of the results. A.N. and S.S. wrote the paper. All authors contributed to the paper and approved the final version.

\section{ADDITIONAL INFORMATION}

Ethics approval and consent to participate The protocol was approved by the Medical Ethics Committee (Medical Ethics Committee of the VU University Medical Centre, Amsterdam) in compliance with the local institutional review board regulations [protocol number: U2017.003]. Patients provided informed consent. The study is performed in accordance with the Declaration of Helsinki.

Consent to publish Not applicable.

Data availability The data generated and analysed for the current investigation are not publicly available, but are available through the corresponding author on reasonable request.

Competing interests The authors declare no competing interests.

Funding information This research project did not receive funding.

Supplementary information is available for this paper at https://doi.org/10.1038/ s41416-020-0888-5.

Note This work is published under the standard license to publish agreement. After 12 months the work will become freely available and the license terms will switch to a Creative Commons Attribution 4.0 International (CC BY 4.0).

Publisher's note Springer Nature remains neutral with regard to jurisdictional claims in published maps and institutional affiliations.

\section{REFERENCES}

1. Liontos, M., Anastasiou, I., Bamias, A. \& Dimopoulos, M. A. DNA damage, tumor mutational load and their impact on immune responses against cancer. Ann. Transl. Med. 4, 264 (2016).

2. Keir, M. E., Butte, M. J., Freeman, G. J. \& Sharpe, A. H. PD-1 and its ligands in tolerance and immunity. Annu. Rev. Immunol. 26, 677-704 (2008).

3. Pardoll, D. M. The blockade of immune checkpoints in cancer immunotherapy. Nat. Rev. Cancer 12, 252-264 (2012).

4. Brahmer, J., Reckamp, K. L., Baas, P., Crino, L., Eberhardt, W. E., Poddubskaya, E. et al. Nivolumab versus docetaxel in advanced squamous-cell non-small-cell lung cancer. N. Engl. J. Med. 373, 123-135 (2015).

5. Borghaei, H., Paz-Ares, L., Horn, L., Spigel, D. R., Steins, M., Ready, N. E. et al. Nivolumab versus docetaxel in advanced nonsquamous non-small-cell lung cancer. N. Engl. J. Med. 373, 1627-1639 (2015).

6. Gettinger, S. N., Horn, L., Gandhi, L., Spigel, D. R., Antonia, S. J., Rizvi, N. A. et al. Overall survival and long-term safety of nivolumab (anti-programmed death 1 antibody, BMS-936558, ONO-4538) in patients with previously treated advanced non-small-cell lung cancer. J. Clin. Oncol. 33, 2004-2012 (2015).

7. Gandara, D. R., Paul, S. M., Kowanetz, M., Schleifman, E., Zou, W., Li, Y. et al. Bloodbased tumor mutational burden as a predictor of clinical benefit in non-small-cell lung cancer patients treated with atezolizumab. Nat. Med. 24, 1441-1448 (2018).

8. Hellmann, M. D., Ciuleanu, T. E., Pluzanski, A., Lee, J. S., Otterson, G. A., AudigierValette, C. et al. Nivolumab plus ipilimumab in lung cancer with a high tumor mutational burden. N. Engl. J. Med. 378, 2093-2104 (2018).

9. Garon, E. B., Rizvi, N. A., Hui, R., Leighl, N., Balmanoukian, A. S., Eder, J. P. et al. Pembrolizumab for the treatment of non-small-cell lung cancer. N. Engl. J. Med. 372, 2018-2028 (2015).

10. Reck, M., Rodriguez-Abreu, D., Robinson, A. G., Hui, R., Csoszi, T., Fulop, A. et al Pembrolizumab versus chemotherapy for PD-L1-positive non-small-cell lung cancer. N. Engl. J. Med. 375, 1823-1833 (2016).

11. Hirsch, F. R., McElhinny, A., Stanforth, D., Ranger-Moore, J., Jansson, M., Kulangara, K. et al. PD-L1 immunohistochemistry assays for lung cancer: results from phase 1 of the blueprint PD-L1 IHC assay comparison project. J. Thorac. Oncol. 12, 208-222 (2017)

12. Teng, M. W., Ngiow, S. F., Ribas, A. \& Smyth, M. J. Classifying cancers based on t-cell infiltration and PD-L1. Cancer Res. 75, 2139-2145 (2015). 
13. Taube, J. M., Klein, A., Brahmer, J. R., Xu, H., Pan, X., Kim, J. H. et al. Association of PD-1, PD-1 ligands, and other features of the tumor immune microenvironment with response to anti-PD-1 therapy. Clin. Cancer Res. 20, 5064-5074 (2014).

14. Tumeh, P. C., Harview, C. L., Yearley, J. H., Shintaku, I. P., Taylor, E. J., Robert, L. et al. PD-1 blockade induces responses by inhibiting adaptive immune resistance. Nature 515, 568-571 (2014).

15. Herbst, R. S., Soria, J. C., Kowanetz, M., Fine, G. D., Hamid, O., Gordon, M. S. et al. Predictive correlates of response to the anti-PD-L1 antibody MPDL3280A in cancer patients. Nature 515, 563-567 (2014).

16. Bubendorf, L., Buttner, R., Al-Dayel, F., Dietel, M., Elmberger, G., Kerr, K. et al. Testing for ROS1 in non-small cell lung cancer: a review with recommendations. Virchows Arch. 469, 489-503 (2016).

17. Thunnissen, E., Allen, T. C., Adam, J., Aisner, D. L., Beasley, M. B., Borczuk, A. C. et al. Immunohistochemistry of pulmonary biomarkers: a perspective from members of the pulmonary pathology society. Arch. Pathol. Lab. Med. 142, 408-419 (2018).

18. Galon, J., Costes, A., Sanchez-Cabo, F., Kirilovsky, A., Mlecnik, B., Lagorce-Pages, C. et al. Type, density, and location of immune cells within human colorectal tumors predict clinical outcome. Science 313, 1960-1964 (2006).

19. Donnem, T., Hald, S. M., Paulsen, E. E., Richardsen, E., Al-Saad, S., Kilvaer, T. K. et al. Stromal CD8+ T-cell density-a promising supplement to TNM staging in nonsmall cell lung cancer. Clin. Cancer Res. 21, 2635-2643 (2015).

20. Paulsen, E. E., Kilvaer, T. K., Khanehkenari, M. R., Al-Saad, S., Hald, S. M., Andersen, S. et al. Assessing PDL-1 and PD-1 in Non-small cell lung cancer: a novel immunoscore approach. Clin. Lung Cancer 18, 220-233 (2017).

21. Eisenhauer, E. A., Therasse, P., Bogaerts, J., Schwartz, L. H., Sargent, D., Ford, R. et al. New response evaluation criteria in solid tumours: revised RECIST guideline (version 1.1). Eur. J. Cancer 45, 228-247 (2009).

22. Mazzaschi, G., Facchinetti, F., Missale, G., Canetti, D., Madeddu, D., Zecca, A. et al. The circulating pool of functionally competent NK and CD8 + cells predicts the outcome of anti-PD1 treatment in advanced NSCLC. Lung Cancer 127, 153-163 (2019).

23. Fumet, J. D., Richard, C., Ledys, F., Klopfenstein, Q., Joubert, P., Routy, B. et al. Prognostic and predictive role of CD8 and PD-L1 determination in lung tumor tissue of patients under anti-PD-1 therapy. Br. J. Cancer 119, 950-960 (2018).

24. Daud, A. I., Loo, K., Pauli, M. L., Sanchez-Rodriguez, R., Sandoval, P. M., Taravati, K. et al. Tumor immune profiling predicts response to anti-PD-1 therapy in human melanoma. J. Clin. Invest. 126, 3447-3452 (2016).

25. Soo, R. A., Chen, Z., Yan Teng, R. S., Tan, H. L., lacopetta, B., Tai, B. C. et al. Prognostic significance of immune cells in non-small cell lung cancer: metaanalysis. Oncotarget 9, 24801-24820 (2018).

26. de Biasi, A. R., Villena-Vargas, J. \& Adusumilli, P. S. Cisplatin-induced antitumor immunomodulation: a review of preclinical and clinical evidence. Clin. Cancer Res. 20, 5384-5391 (2014).

27. Parra, E. R., Villalobos, P., Behrens, C., Jiang, M., Pataer, A., Swisher, S. G. et al. Effect of neoadjuvant chemotherapy on the immune microenvironment in non-small cell lung carcinomas as determined by multiplex immunofluorescence and image analysis approaches. J. Immunother. Cancer 6, 48 (2018).

28. Deng, L., Liang, H., Burnette, B., Beckett, M., Darga, T., Weichselbaum, R. R. et al. Irradiation and anti-PD-L1 treatment synergistically promote antitumor immunity in mice. J. Clin. Invest. 124, 687-695 (2014).
29. Zhang, P., Su, D. M., Liang, M. \& Fu, J. Chemopreventive agents induce programmed death-1-ligand 1 (PD-L1) surface expression in breast cancer cells and promote PD-L1-mediated T cell apoptosis. Mol. Immunol. 45, 1470-1476 (2008).

30. Omori, S., Kenmotsu, H., Abe, M., Watanabe, R., Sugino, T., Kobayashi, H. et al. Changes in programmed death ligand 1 expression in non-small cell lung cancer patients who received anticancer treatments. Int J. Clin. Oncol. 23, 1052-1059 (2018).

31. Herbst, R. S., Baas, P., Perez-Gracia, J. L., Felip, E., Kim, D. W., Han, J. Y. et al. Use of archival versus newly collected tumor samples for assessing PD-L1 expression and overall survival: an updated analysis of KEYNOTE-010 trial. Ann. Oncol. 30, 281-289 (2019).

32. Lin, H., Wei, S., Hurt, E. M., Green, M. D., Zhao, L., Vatan, L. et al. Host expression of PD-L1 determines efficacy of PD-L1 pathway blockade-mediated tumor regression. J. Clin. Invest. 128, 805-815 (2018).

33. Tang, H., Liang, Y., Anders, R. A., Taube, J. M., Qiu, X., Mulgaonkar, A. et al. PD-L1 on host cells is essential for PD-L1 blockade-mediated tumor regression. J. Clin. Invest. 128, 580-588 (2018).

34. Carbone, D. P., Reck, M., Paz-Ares, L., Creelan, B., Horn, L., Steins, M. et al. First-line nivolumab in stage iv or recurrent non-small-cell lung cancer. N. Engl. J. Med. 376, 2415-2426 (2017).

35. Kowanetz, M., Zou, W., Gettinger, S. N., Koeppen, H., Kockx, M., Schmid, P. et al. Differential regulation of PD-L1 expression by immune and tumor cells in NSCLC and the response to treatment with atezolizumab (anti-PD-L1). Proc. Natl Acad. Sci. USA 115, 10119-10126 (2018).

36. Casadevall, D., Clave, S., Taus, A., Hardy-Werbin, M., Rocha, P., Lorenzo, M. et al. Heterogeneity of tumor and immune cell PD-L1 expression and lymphocyte counts in surgical NSCLC samples. Clin. Lung Cancer 18, 682-691 (2017).

37. Gniadek, T. J., Li, Q. K., Tully, E., Chatterjee, S., Nimmagadda, S. \& Gabrielson, E. Heterogeneous expression of PD-L1 in pulmonary squamous cell carcinoma and adenocarcinoma: implications for assessment by small biopsy. Mod. Pathol. 30, 530-538 (2017)

38. Ilie, M., Long-Mira, E., Bence, C., Butori, C., Lassalle, S., Bouhlel, L. et al. Comparative study of the PD-L1 status between surgically resected specimens and matched biopsies of NSCLC patients reveal major discordances: a potential issue for anti-PD-L1 therapeutic strategies. Ann. Oncol. 27, 147-153 (2016).

39. Bigras, G., Mairs, S., Swanson, P. E., Morel, D., Lai, R. \& Izevbaye, I. Small biopsies misclassify up to $35 \%$ of PD-L1 assessments in advanced lung non-small cell lung carcinomas. Appl. Immunohistochem. Mol. Morphol. 26, 701-708 (2018).

40. Freidin, M. B., Bhudia, N., Lim, E., Nicholson, A. G., Cookson, W. O. \& Moffatt, M. F. Impact of collection and storage of lung tumor tissue on whole genome expression profiling. J. Mol. Diagn. 14, 140-148 (2012).

41. van Dongen, G. A., Poot, A. J. \& Vugts, D. J. PET imaging with radiolabeled antibodies and tyrosine kinase inhibitors: immuno-PET and TKI-PET. Tumour Biol. 33, 607-615 (2012).

42. Pelekanou, V., Barlow, W. E., Nahleh, Z. A., Wasserman, B., Lo, Y. C., von Wahlde, M. $\mathrm{K}$. et al. Tumor-infiltrating lymphocytes and PD-L1 expression in pre- and posttreatment breast cancers in the SWOG S0800 phase II neoadjuvant chemotherapy trial. Mol. Cancer Ther. 17, 1324-1331 (2018).

43. Yang, J. H., Kim, H., Roh, S. Y., Lee, M. A., Park, J. M., Lee, H. H. et al. Discordancy and changes in the pattern of programmed death ligand 1 expression before and after platinum-based chemotherapy in metastatic gastric cancer. Gastric Cancer 22, 147-154 (2018). 